\title{
Attalea butyracea próximas a las viviendas como posible fuente de infestación domiciliaria por Rhodnius prolixus (Hemiptera: Reduviidae) en los Llanos Orientales de Colombia
}

\author{
Víctor Manuel Angulo, Lyda Esteban, Katherine Paola Luna \\ Centro de Investigaciones en Enfermedades Tropicales. CINTROP, Universidad Industrial de Santander, \\ Piedecuesta, Colombia
}

Introducción. La presencia de Rhodnius prolixus en palmas en los Llanos Orientales, zona de alto riesgo para la infección por Trypanosoma cruzi, y su frecuente visita al intradomicilio, hacen necesario confirmar este hábitat como posible fuente de infestación.

Objetivo. Comprobar la infestación por $R$. prolixus en Attalea butyracea próximas a viviendas en los Llanos Orientales.

Materiales y métodos. Se examinaron palmas próximas a las viviendas rurales en los departamentos de Casanare y Arauca, utilizando trampas con cebo vivo, y mediante búsqueda activa y vigilancia comunitaria, se recolectaron triatominos en el intradomicilio. Con criterios morfológicos se determinó la especie recolectada y con la técnica de PCR se estableció la infección natural.

Resultados. Se recolectaron 529 ninfas y adultos de $R$. prolixus y 5 ninfas de Eratyrus mucronatus en $111 / 172(64,53 \%)$ A. butyracea ubicadas a menos de $500 \mathrm{~m}$ de las viviendas. En el mismo periodo se recolectaron en el intradomicilio 132 R. prolixus y especímenes de Psammolestes arthuri, Cavernicola pilosa, Triatoma maculata, Panstrongylus geniculatus y 20 ninfas de $E$. mucronatus en un cúmulo de adobes en el corredor de una vivienda. Se detectó infección natural por $T$. cruzi en 28,76\% de $R$. prolixus de palmas y $30 \%$ del intradomicilio.

Conclusiones. Los resultados del presente estudio revelan una nueva situación epidemiológica de transmisión de la enfermedad de Chagas, caracterizada por las altas tasas de infestación de $R$. prolixus en palmas próximas a viviendas y su frecuente intrusión; su impacto debe ser evaluado para diseñar medidas de prevención de la enfermedad.

Palabras clave: Rhodnius, Triatominae, enfermedad de Chagas, Trypanosoma cruzi, Colombia.

Attalea butyracea palms adjacent to housing as a source of infestation by Rhodnius prolixus (Hemiptera: Reduviidae)

Introduction. The presence of Rhodnius prolixus in palms on the eastern plains area of Colombia provide a high risk for infection with Trypanosoma cruzi. Intensive surveillance of the palm tree habitat are necessary to confirm the presence of bugs and provide evidence that this habitat is a source of house infestations.

Objective. The infestation by $R$. prolixus in the palm, Attalea butyracea, was investigated near homes for assessment of risk.

Materials and methods. Palms were examined for Rhodnius bugs adjacent to rural housing in the eastern plains provinces of Casanare and Arauca, Colombia. Bait traps were used as well as an active search by the investigators and a community watch program to collect intradomiciliary triatomine bugs. Collected species were identified by morphological criteria,and natural T.cruzi infections in the triatomine bugs were detected by the PCR technique.

Results. In 111 of 172 (64.5\%) palms (A. butyracea) inspected, 529 nymphs and adults of $R$. prolixus and 5 nymphs of Eratyrus mucronatus were collected. The A. butyracea were located within $500 \mathrm{~m}$ of housing. In the same period, $132 R$. prolixus and specimens of the triatomine species Psammolestes arthuri, Cavernicola pilosa, Triatoma maculata, Panstrongylus geniculatus were collected inside houses. Twenty nymphs of $E$. mucronatus were found in a pile of bricks on the porch of a home. Natural infection of $T$. cruzi was detected in $28.8 \%$ of $R$. prolixus from palm trees and $30.0 \%$ inside houses.

Conclusions. A new epidemiological scenario of transmission of Chagas disease was revealed, characterized by high rates of infestation of $R$. prolixus in palms near houses with frequent intrusion into houses. In designing measures to prevent Chagas disease, the association of palm trees, triatomine bugs and housing proximity is a significant consideration.

Keys words: Rhodnius, Triatominae, Chagas disease, Trypanosoma cruzi, Colombia. 
La enfermedad de Chagas es trasmitida principalmente por insectos triatominos introducidos y adaptados al domicilio humano, como Triatoma infestans en los países del Cono Sur y Rhodnius prolixus en Centroamérica, Colombia y Venezuela. Las acciones de control para estas dos especies han sido efectivas, lo que conllevó a su eliminación en el ambiente doméstico en amplias zonas de Latinoamérica (1). Sin embargo, se ha incrementado la notificación sobre la incursión al intradomicilio humano de especies que ocupan hábitats silvestres de los géneros más importantes, es decir, Rhodnius, Panstrongylus y Triatoma, especialmente R. prolixus en Venezuela y Triatoma dimidiata en Colombia y Centroamérica (2-6).

La colonización y las altas densidades de $R$. prolixus en el intradomicilio han sido características en áreas rurales de la zona oriental, consideradas de gran riesgo para la transmisión de la enfermedad en Colombia (7-10); otras especies, como T. dimidiata, $T$. maculata y $T$. venosa, colonizan las viviendas en menor grado. Como visitante frecuente se ha reportado $P$. geniculatus y, como esporádicos, $R$. pallescens, $R$. pictipes, Eratyrus cuspidatus, $P$. rufotuberculatus, $R$. colombiensis, Panstrogylus humeralis, T. dispar, Belminus herreri, B. corredori y $B$. ferroae (11-15).

Por otro lado, Gamboa en 1963 y otros investigadores en las siguientes tres décadas, encontraron $R$. prolixus, considerado por varias décadas exclusivamente doméstico, en palmas en Venezuela. Su presencia en este hábitat se explicó por el ingreso pasivo de estados inmaduros en las hojas de palmas que se utilizaban para la construcción de los techos (16-19). En Colombia, en 1981 y 1984, D'Alessandro, et al. $(20,21)$, reportaron $R$. prolixus en los Llanos Orientales en varias especies de palmas, incluyendo $A$. butyracea; ellos relacionaron su presencia en estos árboles de abundante follaje con la disponibilidad de huéspedes mamíferos, aves, reptiles y anfibios $(20,21)$. En 2005, Angulo, et al., y Pinto, et al., nuevamente encontraron esta especie en los Llanos Orientales (Angulo

\section{Correspondencia:}

Víctor Manuel Angulo, CINTROP, Universidad Industrial de Santander, km 2 vía Guatiguará, Piedecuesta, Santander, Colombia

Teléfono: (577) 634 4000, extensión 3526; fax: (577) 634 4000, extensión 3571

pitorio@hotmail.com

Recibido: 16/08/11; aceptado:05/03/12
VM, Esteban L. Rhodnius prolixus (Hemiptera: Reduviidae) en palmas asociadas al domicilio humano en los Llanos Orientales de Colombia. Memorias, I Congreso Internacional de Salud en el Trópico. Facultad de Ciencias de la Salud, Villavicencio: 2005, y Pinto N, Marín D, Herrera C, Vallejo G, Naranjo JM, Guhl F. Comprobación del ciclo silvestre de Rhodnius prolixus Stål, en reductos de $A$. butyracea en el departamento del Casanare. Biomédica. 2005;25:159); sin embargo, poco valor se ha dado a estas observaciones en el ciclo de transmisión de la enfermedad en el país.

El hallazgo de altas tasas de infección en los estudios serológicos de donantes y escolares, entre 1997 y 2004, y de infestación en los departamentos de Casanare y Arauca, permitió catalogar esta zona como de alto riesgo para la transmisión de la enfermedad de Chagas (22-24).

Dada la importancia del tema y con el propósito de contribuir a la evaluación del riesgo que las palmas representan como focos silvestres de triatominos en la transmisión de $T$. cruzi a la población humana y animales domésticos en esta zona del país, se presentan los resultados de la infestación de $R$. prolixus en palmas de $A$. butyracea próximas al domicilio en los municipios de Maní y Nunchía (Casanare) y Arauquita (Arauca).

\section{Materiales y métodos}

\section{Área de estudio}

El estudio se llevó a cabo en 2003 a 2006 en áreas rurales de ocho veredas de cuatro municipios de los departamentos de Casanare y Arauca, ubicados en los Llanos Orientales de Colombia entre los 140 y los $230 \mathrm{msnm}$, caracterizadas por tener piso térmico cálido, temperatura media anual de $26-27^{\circ} \mathrm{C}$, precipitación media anual de $1.598 \mathrm{~mm}$, bosque húmedo tropical en Arauca y en los municipios de Maní y Nunchía en Casanare y bosque seco tropical en Arauquita, con una precipitación de $2.500 \mathrm{~mm}$.

En esta región discurren numerosos ríos de la cuenca del río Orinoco y las actividades económicas de mayor importancia son ganadería, agricultura y minería. Su paisaje se caracteriza por extensas sabanas de pastizales, arbustos, cultivos industriales de arroz y palma africana y, además, parches de bosques de palma en las riveras de los ríos, caños, humedales y lagunas; las viviendas se ubican con frecuencia en potreros de pastoreo rodeadas de pequeñas áreas de cultivo y palmas dispersas 0 cerca a bosques aún conservados (figura 1). 


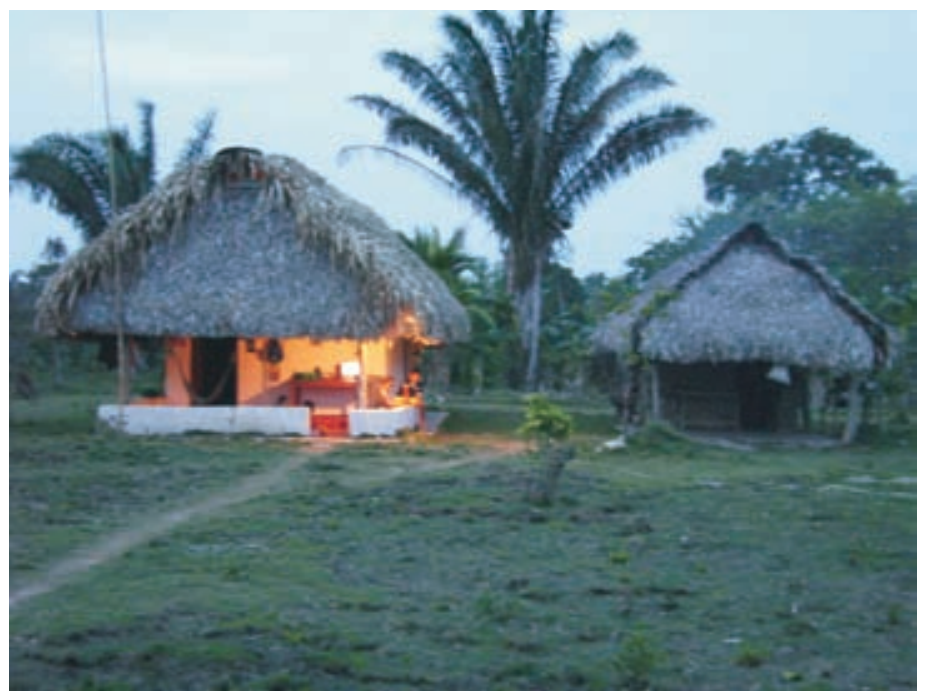

Figura 1. Vivienda con palmas en el peridomicilio, municipio de Maní, departamento de Casanare

Las veredas incluidas se seleccionaron de acuerdo con la confirmación de la presencia de triatominos vectores de la enfermedad de Chagas en el intradomicilio. La búsqueda y recolección de triatominos se hicieron en palmas ubicadas alrededor de las viviendas, incluyendo bosques cercanos (a menos de 500 m), y en el intradomicilio de las viviendas referidas por sus habitantes como visitadas frecuentemente por estos insectos.

\section{Recolección de triatominos en el intradomicilio y estructuras anexas}

Las recolecciones se hicieron mediante la vigilancia entomológica comunitaria, por las familias sensibilizadas y capacitadas en el reconocimiento, búsqueda y manipulación de los insectos, que aceptaron participar en el estudio. Además, se hizo búsqueda hora hombre por parte de los investigadores y técnicos de las secretarías departamentales de salud del Programa Enfermedades Transmitidas por Vectores.

\section{Captura de triatominos en palmas}

Una vez ubicadas las viviendas, se visualizaron y seleccionaron las palmas de $A$. butyracea ubicadas entre 4 y $100 \mathrm{~m}$ alrededor de la vivienda, tanto en los potreros y pequeñas áreas de cultivos como en bosques de palmas de esta especie, ubicados a una distancia entre 20 y 500 m de éstas. Se escogieron palmas adultas cuya corona no sobrepasara una altura de $8 \mathrm{~m}$, para facilitar la colocación de las trampas.

Se colocaron dos tipos de trampas con cebo vivo: la descrita por Noireau en 1999 (25), y una nueva trampa descrita recientemente por Angulo y Esteban, que utiliza un ave como cebo vivo. Estas fueron colocadas simultáneamente en las primeras 42 palmas examinadas para comparar su efectividad. Después de la comparación, se utilizó la que arrojara mejores resultados.

Las trampas fueron izadas y colocadas en la corona (interfoliar) y en los reductos de los raquis de las hojas viejas de las palmas, a una altura entre 4 y $8 \mathrm{~m}$ con ayuda de un tubo de madera obtenido en la zona de 6 a $7 \mathrm{~m}$ de longitud, en el horario de 4 a 6 p.m. Al siguiente día, se retiraron en el horario de 6 a 8 a.m. Se registró la altura de la corona de la palma con respecto al piso, la altura de colocación de la trampa en la palma, y el tipo y el número de cada trampa. Además, se tomaron las coordenadas geográficas de los sitios de muestreo con un dispositivo de GPS (Global Positioning System) (Garmin®).

Los insectos fueron removidos de las trampas en el momento de su retiro de las palmas, colocados en frascos plásticos y rotulados con la información respectiva del lugar, fecha, hora de captura, nombre del jefe de hogar, número de la palma y tipo de trampa, y se empacaron para su transporte al laboratorio del Centro de Investigaciones en Enfermedades Tropicales de la Universidad Industrial de Santander.

\section{Identificación taxonómica}

Se hizo con criterios morfológicos, utilizando las claves de Lent y Wygodzinsky (18) y Carcavallo, et al. (27), ayudados de comparaciones con ejemplares de $R$. prolixus domiciliados de la zona andina y de ejemplares de $R$. robustus de Venezuela. Las ninfas fueron determinadas 
una vez llegaron al estado adulto. Dadas las dificultades para definir el estatus taxonómico de $R$. prolixus de palmas con criterios morfológicos, por su semejanza con $R$. robustus, se seleccionó una submuestra de $R$. prolixus de palmas e intradomicilio para confirmar su identificación taxonómica por estudios moleculares (28).

\section{Determinación de la infestación en palmas}

Se estableció el grado de infestación (palmas infestadas/palmas examinadas), el grado de colonización (palmas con ninfas/palmas infestadas) y el grado de densidad de $R$. prolixus (número de $R$. prolixus recolectados/número de palmas examinadas). Se estableció la estructura por edades de los triatominos recolectados en el intradomicilio, peridomicilio (construcciones anexas) y palmas, y se hizo el cálculo de la razón de momios (odds ratio), para comparar hábitats y áreas geográficas.

\section{Infección natural por Trypanosoma cruzi}

Algunos de los insectos recolectados se disecaron, y su contenido intestinal se extrajo y se observó en microscopio óptico con objetivo de 40X para la búsqueda de tripanosomátidos; parte de la muestra se volvió a suspender en 50 ul de TE (Tris-EDTA, $\mathrm{pH} 8,0$ ) y se almacenó a $-20^{\circ} \mathrm{C}$ para el diagnóstico molecular.

En la obtención de la muestra para la reacción en cadena de la polimerasa (PCR), se siguió el protocolo descrito por Breniere, et al. (29), y se utilizaron los iniciadores S35 y S36 descritos por Sturm, et al. (30), para la amplificación del fragmento de $330 \mathrm{pb}$ de las regiones variables de los minicírculos de T. cruzi.

El volumen final de reacción fue de $20 \mu \mathrm{l}$, que contenía 1,5 mM de $\mathrm{MgCl}$, $50 \mathrm{mM}$ de $\mathrm{KCl}, 10$
$\mathrm{mM}$ deTris- $\mathrm{HCl}, 200 \mu \mathrm{M}$ de dNTPs, 0,2 uM de cada iniciador y $1,0 \cup$ de Taq polimerasa (Promega®). La amplificación se llevó a cabo en un termociclador (MJ Research) a una temperatura inicial de $98^{\circ} \mathrm{C}$ por un minuto, seguida de 35 ciclos $\left(94{ }^{\circ} \mathrm{C}\right.$ por un minuto, $64{ }^{\circ} \mathrm{C}$ por un minuto, $72{ }^{\circ} \mathrm{C}$ por 45 segundos) y un ciclo final a $72{ }^{\circ} \mathrm{C}$ por 10 minutos.

En todos los experimentos se incluyeron controles negativos y controles positivos. Los productos de amplificación fueron visualizados en geles de poliacrilamida coloreados con nitrato de plata.

Se compararon las proporciones de infección natural por la técnica de PCR entre los hábitats y las regiones geográficas, utilizando la prueba de ji al cuadrad, con el programa Epilnfo, versión 6.04d.

\section{Consideraciones éticas}

El estudio se desarrolló dentro del marco de varios proyectos de investigación, ejecutados entre 2002 y 2008, relacionados en el capítulo de financiación y aprobados por el Comité de Ética de la Universidad Industrial de Santander, el cual consideró que "EI protocolo de investigación de este proyecto se aprobó por cumplir las normas éticas vigentes".

\section{Resultados}

\section{Infestación de viviendas y estructuras anexas}

La comunidad reportó la presencia de triatominos en 45 viviendas (cuadro 1), en 20 de las cuales se recolectaron $99 R$. prolixus adultos y 33 ninfas (22 de las ninfas de quinto estadio y 7 de cuarto estadio); además, una hembra de $P$. arthuri y otra de C. pilosa, dos hembras de T. maculata y un macho de $P$. geniculatus. La búsqueda horahombre fue negativa, excepto por 20 ninfas de

Cuadro 1. Infestación de Attalea butyracea por Rhodnius prolixus en Casanare y Arauca

\begin{tabular}{|c|c|c|c|c|c|c|}
\hline Departamento & Municipio & Vereda & $\begin{array}{c}\text { Viviendas con } \\
\text { palmas examinadas }\end{array}$ & $\begin{array}{l}\text { Especímenes } \\
\text { recolectados }\end{array}$ & $\begin{array}{l}\text { A. butyracea positivas/ } \\
\text { total examinadas }\end{array}$ & $\%$ \\
\hline \multirow[t]{5}{*}{ Casanare } & \multirow[t]{3}{*}{ Maní } & Guinea & 6 & 88 & $25 / 35$ & 71,42 \\
\hline & & Llanerita & 8 & 81 & $13 / 19$ & 68,42 \\
\hline & & El Amparo & 4 & 125 & $10 / 10$ & 100 \\
\hline & \multirow[t]{3}{*}{ Nunchía } & La Palmira & 12 & 142 & $35 / 49$ & 71,42 \\
\hline & & Casadero & 2 & 2 & $1 / 1$ & 100 \\
\hline Subtotal & & & 32 & 438 & $84 / 114$ & 73,68 \\
\hline \multirow[t]{3}{*}{ Arauca } & \multirow[t]{2}{*}{ Arauca } & El Torno & 2 & 45 & $11 / 18$ & 61,1 \\
\hline & & La Pastora & 2 & 15 & $6 / 20$ & 30,0 \\
\hline & Arauquita & La Arenosa & 9 & 31 & $10 / 20$ & 50,0 \\
\hline Subtotal & & & 13 & 91 & $27 / 58$ & 46,55 \\
\hline Total & & & 45 & 529 & $111 / 172$ & 64,53 \\
\hline
\end{tabular}


Cuadro 2. Número de triatominos recolectados según el estado de desarrollo en diferentes hábitats en Arauca y Casanare

\begin{tabular}{|c|c|c|c|c|c|c|c|c|c|}
\hline \multirow[b]{2}{*}{ Especie } & \multicolumn{7}{|c|}{ Estados de desarrollo } & \multirow[b]{2}{*}{ Total } & \multirow[b]{2}{*}{ Hábitat } \\
\hline & n1 & $\mathrm{n} 2$ & n3 & n4 & n5 & Hembras & Machos & & \\
\hline R. prolixus & 51 & 84 & 145 & 94 & 102 & 28 & 25 & 529 & A. butyracea \\
\hline R. prolixus & 1 & 2 & 1 & 7 & 22 & 55 & 44 & 132 & Intradomicilio \\
\hline R. prolixus & 0 & 0 & 0 & 2 & 3 & 3 & 2 & 10 & Gallinero \\
\hline E. mucronatus & 0 & 1 & 3 & 1 & 0 & 0 & 0 & 5 & A. butyracea \\
\hline E. mucronatus & 0 & 0 & 4 & 13 & 3 & 0 & 0 & 20 & Intradomicilio \\
\hline T. maculata & 0 & 0 & 4 & 16 & 24 & 0 & 0 & 44 & Gallinero \\
\hline T. maculata & 0 & 0 & 0 & 0 & 0 & 2 & 0 & 2 & Intradomicilio \\
\hline P. arthuri & 0 & 0 & 0 & 0 & 0 & 1 & 0 & 1 & Intradomicilio \\
\hline C. pilosa & 0 & 0 & 0 & 0 & 0 & 1 & 0 & 1 & Intradomicilio \\
\hline$P$. geniculatus & 0 & 0 & 0 & 0 & 0 & 0 & 1 & 1 & Intradomicilio \\
\hline
\end{tabular}

E. mucronatus encontradas en un cúmulo de adobes en el corredor de una vivienda. Los técnicos del Programa de Enfermedades Transmitidas por Vectores recolectaron $44 T$. maculata en gallineros de dos viviendas (cuadro 2).

\section{Infestación de palmas}

En Casanare se detectó infestación por $R$. prolixus en 84 de 114 palmas $\left(73,7 \%\right.$; $\left.I_{95 \%} 64,5-81,3\right)$, y en Arauca, en 27 de 58 palmas (46,6 \%; $I C_{5 \%}$ $33,5-60,0$ ) (cuadro 1). La razón de momios fue 3,2 $\left(\mathrm{IC}_{95 \%} 1,6-6,6\right)(\mathrm{p}<0,05)$ en Casanare comparado con Arauca, lo que indica un mayor y significativo riesgo para la presencia de $R$. prolixus en las palmas de Casanare. Los índices de colonización fueron de $100 \%(27 / 27)$ y de $94,4 \%(79 / 84)$ en palmas de Arauca y de Casanare, respectivamente ( $p>0,05$ ). Los grados de densidad fueron $3,37 \%$ $(91 / 27)$ en Arauca y $5,16 \%$ (435/84) en Casanare $(p>0,05)$.

\section{Palmas revisadas}

Se examinaron entre 5 y 6 palmas de $A$. butyracea por noche durante 32 noches, para un total de 172 palmas (114 en Casanare y 58 en Arauca) alrededor de 45 viviendas (32 en Casanare y 13 en Arauca). Se recolectaron 534 triatominos, de los cuales, 99,1 $\%(529 / 534)$ correspondieron a $R$. prolixus (438 en Casanare y 91 en Arauca), y 0,9\%, (5/534) a $E$. mucronatus en Casanare (cuadro 1).

\section{Distribución de triatominos por edades}

Según el grado de desarrollo de las ninfas (n1 a n5), en palmas se encontró $R$. prolixus distribuidos así: 51 (9,64\%) n1; 84 (15,88\%) n2; 145 (27,41\%) n3; 94 (17,77\%) n4; y $102(19,28 \%)$ n5; hubo 28 $(5,29 \%)$ hembras y $25(4,73 \%)$ machos; hubo 90 $\%$ de estados inmaduros y $10 \%$ de adultos. Por el contrario, en el intradomicilio se distribuyeron así:
$1(0,76 \%)$ n1; 2 (1,52\%) n2; 1 (0,76 \%) n3; 7 (5,30 \%) n4; y 22 (16,67\%) n5; hubo 55 (41,67\%) hembras y 44 (33,33\%) machos; hubo $25 \%$ de estados inmaduros y $75 \%$ de adultos.

Al comparar la distribución de adultos y estados inmaduros entre palmas e intradomicilio, se observaron diferencias significativas $(p<0,05)$. Se recolectaron, también, dos ninfas 1 , tres ninfas 3 y una ninfa 4 de $E$. mucronatus, y solo adultos de $P$. arthuri, C. pilosa, T. maculata y $P$. geniculatus (cuadro 2).

\section{Infección natural por Trypanosoma cruzi}

Se observaron tripanosomátidos en el examen directo de heces en $24,42 \%$ (32/131) triatominos, $31,08 \%(23 / 74)$ de los recolectados en palmas y $15,78 \%(9 / 57)$ del intradomicilio $(p<0,05)$. La infección por T. cruzi mediante PCR fue de 29,2\% (33/111), y de $28,76 \%(21 / 73)$ y $30 \%(12 / 40)$ en palmas e intradomicilio, respectivamente $(p>0,05)$. En Casanare resultó positiva en 23,8 \% (20/84), y en Arauca, en 44,22 \% (13/29) $(p<0,05)$. En los insectos de palmas de Casanare, hubo infección en $22,4 \%$ (11/48), y de Arauca, en 41,7\% (10/24), con una razón de momios de $2,4\left(\mathrm{IC}_{95 \%} 0,76-8,08\right)$ $(p>0,05)$. En los triatominos del intradomicilio de Casanare, hubo infección en 25,7\% (9/35), y de Arauca, en $60 \%(3 / 5)$, con una razón de momios de 4,33 ( $\left.\mathrm{IC}_{95 \%} 0,47-45,97\right)(\mathrm{p}>0,05)$.

\section{Uso de las trampas}

En la comparación inicial de la frecuencia de resultados positivos de las 42 trampas, la razón de momios fue $2,7 \quad\left(\mathrm{IC}_{95 \%}, 0,57-13,27\right)$ ( $\left.\mathrm{p}>0,05\right)$. Hubo 15 coincidencias positivas y 10 negativas; hubo 4 resultados positivos con cebo de ratón y 14 con cebo de ave. Con trampas de Noireau se recolectaron 62 triatominos $y$, con trampas de 
Angulo y Esteban, $116(p<0,05)$. El número máximo de insectos por trampa fue 12 con las primeras y 22 con las segundas.

\section{Discusión}

Los altos grados de infestación y colonización de $A$. butyracea por $R$. prolixus en el peridomicilio de las áreas rurales de municipios de Casanare y Arauca, confirman esta especie de palma como hábitat natural de $R$. prolixus en la zona geográfica estudiada y corroboran lo reportado previamente en Colombia y Venezuela $(16,21,31)$.

Las diferencias en la distribución por edades en cada hábitat fue semejante a lo hallado por SuarezDávalos, et al. (32), para $R$. ecuadoriensis y Romaña, et al. (33), para $R$. pallescens. Según sus hallazgos, una mayor y significativa proporción de estadios juveniles en palmas podría indicar que las poblaciones silvestres de estas especies están bien establecidas y se reproducen en este hábitat (32), lo que sugiere en términos eco-epidemiológicos que las palmas pueden ser consideradas el principal ecótopo silvestre de estas poblaciones, y un marcador ecológico para identificar potencialmente la presencia de triatominos vectores y reservorios del ciclo selvático de T. cruzi (33).

La recolección de especímenes principalmente adultos en las viviendas, sugiere la migración de estos insectos desde las palmas próximas al domicilio. Este fenómeno de intrusión se ha descrito para varias especies de triatominos en otros países, incluyendo Colombia y, además, se ha comprobado experimentalmente $(2,21,34-36)$. La hipótesis de la infestación de las viviendas mediante transporte pasivo de insectos en las hojas de palma llevadas a la vivienda para construir o renovar el techo, no fue reportado por los moradores de esta zona en este estudio (37).

Entre las principales causas de la domiciliación y el consecuente aumento del riesgo de transmisión de T. cruzi al hombre, se han señalado: la disminución de refugios y huéspedes de triatominos silvestres como resultado de la expoliación del bosque; la colonización humana junto con la mala calidad de la vivienda, y el efecto de la atracción de los triatominos por la luz artificial de las viviendas durante la noche $(5,21,38-43)$. Esto último fue manifestado por los habitantes de esta zona durante el estudio. Las fumigaciones aéreas para la erradicación de los cultivos ilícitos podrían explicar las tasas de infestación significativamente menores en Arauca.
Attalea butyracea es la especie de mayor dispersiónen Centroamérica, Colombia, Venezuela y parte de Ecuador (44), donde se ha registrado la mayor densidad de triatominos, especialmente del género Rhodnius, y su distribución en Colombia (por debajo de los $1.800 \mathrm{msnm}$ ) coincide con las áreas endémicas para la enfermedad de Chagas (45). Lo anterior nos obliga a tener en cuenta la distribución de $A$. butyracea para identificar las zonas de riesgo de intrusión de $R$. prolixus u otros triatominos vectores de la enfermedad de Chagas a las viviendas y evaluar el impacto que esto tiene en la transmisión de la infección por $T$. cruzi. La conservación de las palmas en los potreros de pastoreo de ganado, zonas de cultivos o bosques alrededor de las viviendas, hace parte del paisaje típico de extensas áreas de los Llanos Orientales de Colombia, pero, según lo observado en este estudio y en otros estudios preliminares (46-47), representa riesgo de infestación domiciliaria de insectos vectores del parásito $T$. cruzi, agente causal de la enfermedad de Chagas. Lo anterior no sugiere la destrucción de estos ecótopos naturales como medida de control de la intrusión, sino la búsqueda de alternativas protectoras de las poblaciones humanas expuestas.

El paisaje antrópico descrito en este documento constituye un núcleo enzoótico sinantrópico, donde los animales silvestres oportunistas, como marsupiales y cánidos, se concentran en las palmas próximas a las viviendas atraídos por productos vegetales, desechos de productos alimentarios y animales domésticos potenciales presas. La disponibilidad de huéspedes posiblemente beneficia la concentración de triatominos en estas palmas al ofrecer alimento y refugio y la intrusión de éstos a las viviendas, lo cual incrementa el riesgo de transmisión de $T$. cruzi dado el carácter ecléctico de R. prolixus.

La movilidad de las poblaciones de $R$. prolixus entre hábitats ha sido demostrada en estudios epidemiológicos con el uso herramientas morfológicas, serológicas y moleculares, en Venezuela (48) y Colombia (28) (Farfán AE, Esteban L, Angulo VM. Hospederos de $R$. prolixus del domicilio y palmas en los Llanos Orientales de Colombia. Boletín de Malariología y Salud Ambiental. 2007; 47(Suppl.1):244; Angulo VM, Esteban L. Evidencias morfológicas del proceso de adaptación de $R$. prolixus Stål (Hemiptera: Reduviidae) silvestres hacia los domicilios humanos en los llanos orientales de Colombia. Boletín de Malariología y Salud Ambiental. 2007; 47(Suppl.1):247-8). 
Las tasa de infección global por $T$. cruzi de los insectos procesados en el estudio fue similar a la reportada por Carcavallo, et al. (31), en palmas y por Angulo, et al. (34), en poblaciones domiciliarias de $R$. prolixus en el área andina en Colombia. La tasa de infección en Arauca podría sugerir un mayor contacto con reservorios infectados concentrados en las palmas próximas a las viviendas, reducidas en número después de la deforestación.

El hallazgo de T. maculata, P. arthuri, C. pilosa, $P$. geniculatus y $E$. mucronatus en las viviendas (esta última también en palmas), revela un comportamiento similar al de $R$. prolixus, aunque en menor proporción.

La efectividad demostrada por los dos tipos de trampas utilizadas para detectar la infestación de las palmas, ofrece una alternativa para abandonar otros métodos de mayor riesgo para los investigadores en el examen de palmas y menos amigables con el medio ambiente. Además, los resultados mostraron que la trampa de Angulo-Esteban ensayada en este estudio (26) puede utilizarse para recolectar mayor cantidad de triatominos vivos que la trampa descrita por Noireau (25).

Los resultados de este estudio y los datos presentados por Luna (28), según los cuales las poblaciones de $R$. prolixus encontradas en domicilios y palmas se comportan como una población panmíctica, revelan una nueva situación epidemiológica de la enfermedad de Chagas que ya fue descrito en Venezuela y que deberá ser valorado en Colombia $(48,49)$.

Por consiguiente, como lo han recomendado varios autores (50-52), es necesario continuar elaborando trabajos para establecer y evaluar el impacto de esta nueva situación caracterizada por las altas tasas de infestación de $R$. prolixus en palmas próximas a viviendas y su frecuente intrusión, con el fin de orientar el diseño adecuado de estrategias de prevención y control que disminuyan el riesgo de contacto de las poblaciones humanas con $R$. prolixus silvestre.

\section{Agradecimientos}

Al señor Bayardo Rincón, directivos y personal del Programa de Enfermedades Transmitidas por Vectores de la Secretaría de Salud de Casanare; al señor Germán Gómez, directivos y personal del Programa de Enfermedades Transmitidas por Vectores de la Secretaría de Salud de Arauca; a las comunidades de las áreas rurales de las zonas estudiadas.

\section{Conflicto de intereses}

Los autores del presente escrito declaran que no existe conflicto de intereses.

\section{Financiación}

Este trabajo fue financiado por Colciencias, proyecto No 1102-04-13010, 1102-04-13029 y recibió apoyo logístico de las secretarías de salud de Casanare y Arauca.

\section{Referencias}

1. Dias JCP, Silveira AC, Schofield CJ. The impact of Chagas disease control in Latin America. A review. Mem Inst Oswaldo Cruz. 2002;97:603-12.

2. Lainson JJ, Shaw HF, Fraiha H, Miles MA, Draper CC. Chaga's disease in the Amazon Basin I. Trypanosoma cruzi infections in sylvatic mammals, triatomine bugs and man, in the state of Pará, north Brazil. Trans R Soc Trop Med Hyg. 1979;73:193-204.

3. Dujardin JP, Garcia-Zapata MT, Jurberg J, Roelants P, Cardozo L, Panzera F, et al. Which species of Rhodnius is invading houses in Brazil? Trans R Soc Trop Med Hyg. $1991 ; 85: 679-80$.

4. Angulo VM. Comportamiento de Triatoma dimidiata: un reto para su control. Biomédica. 2005;25(Supl.1):80-2.

5. Dias de Lima AG, Sherlock I. Sylvatic vectors invading houses and the risk of emergence of cases of Chagas disease in Salvador, state of Bahia, Northeast Brazil. Mem Inst Oswaldo Cruz. 2000;95:611-3.

6. CostaJ.The synanthropic process of Chagas disease vectors in Brazil, with special attention to Triatoma brasiliensis Neiva, 1911 (Hemiptera: Reduviidae: Triatominae) population, genetical, ecological, and epidemiological aspects. Mem Inst Oswaldo Cruz. 1999;94(Suppl.1):239-41.

7. Corredor A, Santacruz M, Páez S, Guatame LA. Distribución de los triatominos domiciliados en Colombia. Bogotá: Ministerio de Salud- Instituto Nacional de Salud; 1990. p. 44.

8. Angulo VM, Tarazona Z, Reyes A, Gutiérrez R, Sandoval CM. Ministerio de Salud, Programa Nacional de Prevención y Control de la Enfermedad de Chagas y la Cardiopatía Infantil. En: Angulo VM, editor. Curso-taller Internacional Control y Manejo de la Tripanosomiasis Americana. Bucaramanga: Gráficas Trijaimes; 1999. p. 99-108.

9. Guhl F, Angulo VM, Restrepo M, Nicholls S, Montoya R. Estado del arte de la enfermedad de Chagas en Colombia y estrategias de control. Biomédica. 2003;23(Suppl.1):31-7.

10. Sandoval CM, Gutiérrez R, Luna S, Esteban L, Ariza H, Angulo VM. High density of Rhodnius prolixus in a rural house in Colombia. Trans R Soc Trop Med Hyg. 2000;94:372-3

11. Guhl F, Aguilera G, Pinto N, Vergara D. Distribución geográfica de las especies de triatominos en los departamentos endémicos para la enfermedad de Chagas en Colombia. En: Guhl F, editor. Primer Taller Internacional sobre Control de la Enfermedad de Chagas, Curso de Diagnóstico, Manejo y Tratamiento de la Enfermedad de 
Chagas, VI Reunión de la Iniciativa Andina para el Control de la Enfermedad de Chagas. Bogotá: Ediciones Uniandes; 2005. p. 25-41.

12. Agudelo LA. Experiencias epidemiológicas del Grupo de Chagas de la Universidad de Antioquia. En: Jaramillo N, Parra GJ, Triana O, editores. VIII Curso Internacional de Eco-Epidemiología de la Enfermedad de Chagas y Métodos para su Estudio. Medellín: Ediciones CES; 2005. p. 11323.

13. Sandoval CM, Duarte R, Gutiérrez R, Da Silva-Rocha D, Angulo VM, Esteban L, et al. Feeding sources and natural infection of Belminus herreri (Hemiptera: Reduviidae: Triatominae) from dwellings in Cesar, Colombia. Mem Inst Oswaldo Cruz. 2004;99:137-40.

14. Galvão C, Angulo VM. Belminus corredori, a new species of Bolboderini (Hemiptera: Reduviidae: Triatominae) from Santander, Colombia. Zootaxa. 2006;1241:61-8.

15. Sandoval CM, Pabón E, Jurberg J, Galvão K. Belminus ferroae n. sp. from the Colombian north-east, with a key to the species of the genus (Hemiptera: Reduviidae: Triatominae). Zootaxa. 2007;1443:55-64.

16. Gamboa CJ. Comprobación de Rhodnius prolixus extradomiciliarios en Venezuela. Bol Ofic Sanit Panam.1963;54:18-25.

17. Feliciangeli MD, Torrealba JW. Observaciones sobre Rhodnius prolixus (Hemiptera: Reduviidae) en su biotopo silvestre Copernicia tectorum. Bol Dir Malariol San Amb.1977;XVII:198-205.

18. Lent $\mathbf{H}$, Wygodzinsky P. Revision of the Triatominae: Hemiptera: Reduviidae and their significance as vectors of Chagas disease. Bull Am Mus Nat Hist.1979;163:123-520.

19. Carcavallo RU, Galíndez-Girón I, Jurberg J, Lent H. Atlas of Chagas disease vectors in the Americas. Rio de Janeiro: Editora Fiocruz; 1998. p. 561-600.

20. D'Alessandro A, Barreto P, Thomas M. Nuevos registros de triatominos domiciliarios y extradomiciliarios en Colombia. Colombia Médica.1981;12:75-85.

21. D'Alessandro $\mathbf{A}$, Barreto $\mathbf{P}$, Saravia $\mathbf{N}$, Barreto $\mathbf{M}$. Epidemiology of Trypanosoma cruzi in the oriental plains of Colombia. Am J Trop Med Hyg.1984;33:1084-95.

22. Moncayo A. Chagas disease: Current epidemiological trends after the interruption of vectorial and transfusional transmission in the southern cone countries. Mem Inst Oswaldo Cruz. 2003;98:577-91.

23. Beltrán M, Bermúdez MI, Forero MC, Ayala M, Rodríguez M. Control de la enfermedad de Chagas transfusional en Colombia. En: Guhl F, editor. Primer Taller Internacional sobre Control de la Enfermedad de Chagas, Curso de Diagnóstico, Manejo y Tratamiento de la Enfermedad de Chagas, VI Reunión de la Iniciativa Andina para el Control de la Enfermedad de Chagas. Bogotá: Ediciones Uniandes; 2005. p. 81-5.

24. Niño PA, Robinson A, Tabares R. Situación de la enfermedad de Chagas en el departamento de Arauca. En: Guhl F, editor. Primer Taller Internacional sobre Control de la Enfermedad de Chagas, Curso de Diagnóstico, Manejo y Tratamiento de la Enfermedad de Chagas, VI Reunión de la Iniciativa Andina para el Control de la Enfermedad de Chagas. Bogotá: Ediciones Uniandes; 2005. p. 141- 8.
25. Noireau F, Flores R, Vargas F. Trapping sylvatic Triatominae (Reduviidae) in hollow trees. Trans R Soc Trop Med Hyg. 1999;93:13-4.

26. Angulo VM, Esteban L. Nueva trampa para la captura de triatominos en hábitats silvestres y peri-domésticos. Biomédica. 2011;31:264-8.

27. Carcavallo RU, Galíndez-Girón I, Jurberg J, Lent H. Atlas of Chagas disease vectors in the Américas. Rio de Janeiro: Editora Fiocruz; 1998. p. 107-244.

28. Luna KP. Caracterización genética de poblaciones de Rhodnius prolixus y Triatoma dimidiata en áreas endémicas de Colombia (tesis). Bucaramanga: Universidad Industrial de Santander; 2009.

29. Breniere SF, Bosseno MF, Telleria J, Carrasco R, Vargas $\mathrm{F}$, Yaksic N, et al. Field application of polymerase chain reaction diagnosis and strain typing of Trypanosoma cruzi in Bolivian triatomines. Am J Trop Med Hyg. 1995;53:179-84.

30. Sturmn NR, Degrave W, Morel CM, Simpson L. Sensitive detection and schizodeme classification of Trypanosoma cruzi by amplification of kinetoplast minicircle DNA sequences: Use in diagnosis of Chagas disease. Mol Biochem Parasitol. 1989;33:205-14.

31. CarcavalloR, TonnR, Ortega R, BetancourtP, Carrasquero B. Notas sobre la biología, ecología y distribución geográfica de Rhodnius prolixus Stål, 1859 (Hemiptera: Reduviidae). Bol Dir Malariol San Amb.1978;18:175-98.

32. Suárez-Dávalos V, Dangles 0 , Villacis A, Grijalva MJ. Microdistribution of sylvatic triatomine populations in centralcoastal Ecuador. J Med Entomol. 2010;47:80-8.

33. Romaña CA, Pizarro JC, Rodas E, Guilbert E. Palm trees as ecological indicators of risk areas for Chagas disease. Trans R Soc Trop Med Hyg. 1999;93:594-5.

34. Angulo VM, Gutiérrez R, Rubio I, Joya M, Arismendi MJ, Esteban L, et al. Triatominos domiciliados y silvestres: impacto en la transmisión de la enfermedad de Chagas en Santander. En: Angulo VM, editor. Curso-Taller Internacional Control y Manejo de la Tripanosomiasis Americana. Bucaramanga: Gráficas Trijaimes; 1999. p. 72-6.

35. Gómez-Núñez JC. Resting places, dispersal and survival of $\mathrm{CO}_{60}$ tagged adult Rhodnius prolixus. J Med Entomol. 1969;6:83-6.

36. Texeira AR, Monteiro PS, Rebelo JM, Argañaraz ER, Vieira D, Lauria-Pires L, et al. Emerging Chagas disease: Trophic network and cycle of transmission of Trypanosoma cruzi from palm trees in the Amazon. Emerg Infect Dis. 2001;7:100-12.

37. Gamboa J. Ecología de la tripanosomiasis americana (enfermedad de Chagas) en Venezuela. Bol Inf Dir Malariol San Amb. 1974;14:3-20.

38. D'Ascoli A, Gómez JC. Notas sobre los medios de dispersión del Rhodnius prolixus Stål. Acta Cient Ven. 1966;17:22-5.

39. Pinto J, Borges R. Aspectos sociais da doença de Chagas. Rev Goiana Med. 1979;25:157-68.

40. Forattini OP, Ferreira OA, da Rocha e Silva EO, Rabello EX. Aspectos ecológicos da tripanossomiase Americana XII Variação regional da tendência de Panstrongylus megistus à domiciliação. Rev Saude Publica. 1978;12:209-33. 
41. Gaunt M, Miles M. The ecotopes and evolution of Triatomine bugs (Triatominae) and their associated Trypanosomes. Mem Inst Oswaldo Cruz. 2000;95:557-65.

42. Minoli SA, Lazzari CR. Take-off activity and orientation of triatomines (Heteroptera: Reduviidae) in relation to the presence of artificial lights. Acta Trop. 2006;97:324-30.

43. Castro MC, Barrett TV, Santos WS, Abad-Franch F, Rafael JA. Attraction of Chagas disease vectors (Triatominae) to artificial light sources in the canopy of primary Amazon rainforest. Mem Inst Oswaldo Cruz. 2010;105:1061-4.

44. Henderson A, Galeano G, Bernal R. Field guide to the palms of the Americas. Princeton: Princeton University Press; 1995. p. 352.

45. Vallejo GA, Lozano LE, Carranza JC, Sánchez JL, Jaramillo JC, Guhl F, et al. Ecología de los triatominos no domiciliados en Colombia con especial referencia a $R$. colombiensis en el departamento del Tolima. En: Vallejo G, Carranza, Jaramillo JC, editores. Curso Taller Internacional Biología, epidemiología y control de la Tripanosomosis americana y Lehismaniosis. Ibagué: Lito-ediciones Tolima; 2000. p. 22-8.

46. Whitlaw JT, Chaniotis BN. Palm trees and Chagas' disease in Panama. Am J Trop Med Hyg. 1978;27:873-81.
47. Sánchez- Martín MJ, Feliciangeli MD, CampbellLemdrum D, Davies CR. Could the Chagas disease elimination programme in Venezuela be compromised by reinvasion of houses by sylvatic Rhodnius prolixus bug populations? Trop Med Int Health. 2006;11:1585-93.

48. Feliciangeli MD, Sánchez-Martín M, Marrero R, Davies C, Dujardin JP. Morphometric evidence for a possible role of Rhodnius prolixus from palm trees in house re-infestation in the state of Barinas (Venezuela). Acta Trop. 2007;101:16977.

49. Fitzpatrick S, Feliciangeli MD, Sánchez-Martín MJ, Monteiro FA, Miles MA. Molecular genetics reveal that sylvatic Rhodnius prolixus do colonise rural houses. PLoS Negl Trop Dis. 2008;2:e210.

50. Esteban L, Angulo VM, Feliciangeli MD, Catalá S. Analysis of antennal sensilla patterns of Rhodnius prolixus from Colombia and Venezuela. Mem Inst Oswaldo Cruz. 2005;100:909-14

51. Aguilar HM, Abad-Franch F, Pinto Dias JC, Junqueira AC, Coura JR. Chagas disease in the Amazon Region. Mem Inst Oswaldo Cruz. 2007;102:47-55.

52. Guhl F, Pinto N, Aguilera G. Sylvatic triatominae: A new challenge in vector control transmission. Mem Ins Oswaldo Cruz. 2009;104(Suppl.1):71-5. 